\title{
Development of a fast and easy method for Escherichia coli genome editing with CRISPR/ Cas9
}

\author{
Dongdong Zhao ${ }^{1,2}$, Shenli Yuan ${ }^{1,2}$, Bin Xiong ${ }^{1,2}$, Hongnian Sun ${ }^{1,2}$, Lijun Ye ${ }^{1,2}$, Jing Li ${ }^{1,2}$, Xueli Zhang ${ }^{1,2^{*}}$ \\ and Changhao $\mathrm{Bi}^{1,2^{*}}$ (D)
}

\begin{abstract}
Background: Microbial genome editing is a powerful tool to modify chromosome in way of deletion, insertion or replacement, which is one of the most important techniques in metabolic engineering research. The emergence of CRISPR/Cas9 technique inspires various genomic editing methods.

Results: In this research, the goal of development of a fast and easy method for Escherichia coli genome editing with high efficiency is pursued. For this purpose, we designed modular plasmid assembly strategy, compared effects of different length of homologous arms for recombination, and tested different sets of recombinases. The final technique we developed only requires one plasmid construction and one transformation of practice to edit a genomic locus with 3 days and minimal lab work. In addition, the single temperature sensitive plasmid is easy to eliminate for another round of editing. Especially, process of the modularized editing plasmid construction only takes $4 \mathrm{~h}$.
\end{abstract}

Conclusion: In this study, we developed a fast and easy genome editing procedure based on CRISPR/Cas9 system that only required the work of one plasmid construction and one transformation, which allowed modification of a chromosome locus within 3 days and could be performed continuously for multiple loci.

Keywords: CRISPR/Cas9, E. coli, Genome editing

\section{Background}

Microbial genome editing is for site-specific chromosome modification, which is one of the most useful techniques in research and plays an important role in metabolic engineering and molecular biology research. Current methods include homologous recombinationbased gene targeting [1-3], zinc finger nucleases (ZFNs) [4] and transcription activator-like effector nucleases (TALEN) [5, 6]. However, these techniques are often inefficient, time consuming, laborious, or expensive to use. Thus, a rapid and simple method capable of performing

\footnotetext{
*Correspondence: zhang_xl@tib.cas.cn; bi_ch@tib.cas.cn

${ }^{2}$ Key Laboratory of Systems Microbial Biotechnology, Tianjin Institute of Industrial Biotechnology, Chinese Academy of Sciences, 32 West 7th Ave, Tianjin Airport Economic Park, Tianjin 300308, China

Full list of author information is available at the end of the article
}

precise genome editing with minimal cost remains to be desired.

The clustered regularly interspaced short palindromic repeats (CRISPR)/CRISPR-associated protein (Cas) system is an RNA-guided immune system in many bacteria [7]. This system is able to recognize and generate doublestrand breaks (DSBs) at target sequence. Following the DSB event, native DNA repair will be initiated through non-homologous end joining or homologous recombination in the presence of an exogenous homologous donor sequence [8]. The only restriction for designing a CRISPR/Cas9 guiding sequence is need for a protospacer adjacent motif (PAM) close to genomic target site [9]. The typeII CRISPR/Cas9 system of Streptococcus pyogenes, which requires a mature CRISPR RNA (crRNA), a trans-activating crRNA (tracrRNA) and a DNA endonuclease Cas9 has attracted significant attention and been harnessed for targeted genome editing in different 
organism, both Eukaryotes and Prokaryotes, such as $E$. coli [10-12], human [13], mice [14] and zebrafish [15]. Thus far, the CRISPR-Cas9 system is a powerful and revolutionary tool for genome editing.

The CRISPR/Cas9 system has been widely used for eukaryotic genomes but its applications in bacterial genomes were less studied. CRISPR/Cas9 system was firstly exploited for bacterial genomic editing by Jiang et al., the work was a huge breakthrough for microbial genomic editing, however, high editing efficiency in $E$. coli was not achieved [10]. Recently, researchers achieved very good results in development of Cas9 based gnomic editing techniques $[10-12,16]$. While these methods improved the genome editing techniques, some aspects could be further improved, such as editing efficiency, lab hours, convenience of usage and standard protocols. To tackle these problems, we started the work of development of a simple and fast method for bacterial genome editing with high efficiency. For this purpose, multi-plasmid system was experimented and found to be relatively laborious and time consuming. Low editing efficiency was observed with the plasmid/linear DNA system, especially with wild type and $E$. coli cell factory strains. This was probably due to their strong restriction systems which degrade the linear donor DNAs before integration into chromosome [17]. To avoid these drawbacks, our strategy was designed to edit genome with only one plasmid, which carried all functional parts to make the technique very easy and fast to practice and achieve high efficiency.

\section{Methods}

\section{Bacterial cultivation}

Escherichia coli MG1655 and $\mathrm{DH}_{5} \alpha$ were grown at $30{ }^{\circ} \mathrm{C}$ in lysogeny broth $(\mathrm{LB}, 1 \%(\mathrm{w} / \mathrm{v})$ tryptone, $0.5 \%(\mathrm{w} / \mathrm{v})$ yeast extract, $1 \%(\mathrm{w} / \mathrm{v}) \mathrm{NaCl})$. Kanamycin $(50 \mathrm{mg} / \mathrm{L}$ for E. coli) and Ampicillin (100 mg/L for E. coli) were added to the medium as appropriate. $1 \%(\mathrm{w} / \mathrm{v})$ glucose was added to the culture for Cas9 repression and L-arabinose ( $2 \mathrm{~g} / \mathrm{L}$ final concentration) was used for Cas9 induction when necessary. IPTG and X-gal for blue/white selection were added at concentrations of $0.1 \mathrm{mM}$ and $40 \mu \mathrm{g} / \mathrm{mL}$, respectively [12].

Escherichia coli MG1655 were grown in $5 \mathrm{~mL}$ LB cultures at $30{ }^{\circ} \mathrm{C}$ to an OD600 of 0.6 and then made electrocompetent by concentrating 100 -fold and washing three times with ice-cold 10\% glycerol. 10-100 ng of PCR target plasmid was used for electroporation, shocked cells were added to $1 \mathrm{~mL} \mathrm{LB}$, incubated $1 \mathrm{~h}$ at $30{ }^{\circ} \mathrm{C}$, and then plated on LB petri dishes carrying appropriate antibiotics.

\section{Plasmid construction}

The Cas9 gene from Cas9 expression plasmid (Addgene reference number: 42876), gRNA without N20 (5'G T T T TAGA GCTAGAAATAGCAAG T TAAAA TAAGGCTAGTCCGTTATCAACTTGAAAAAGTGGC ACCGAGTCGGTGCTT-3') and its promoter (5'CTAGGTTTATACATAGGCGAGTACTCTGTTA TGGAGTCAGATCT-3') which were all directly synthetized. pKD46 modular (contains: exo, bet, gam, rep and arabinose operon) was used for plasmid construction as backbone.

pCas9 series and pRed_Cas9 series other than pRed Cas9_recA__poxb41 and pRed_Cas9_recA__lacZ41 were assembled with the Gibson method, of which DNA oligo primers were designed with $\mathrm{j} 5$ and Device Editor [18]. DNA templates were PCR-amplified with Phusion polymerase (NEB) or directly synthesized. PCR products were gel purified, digested with DpnI before Gibson amplification.

For modular design, the pRed_Cas9_recA_Lpoxb300 plasmid was used as template for the PCR amplification of target modules. Homologous arms of $\mathrm{H} 1$ and $\mathrm{H} 2$ were obtained from genomic DNA of E. coli MG1655. pRed Cas9_recA_spoxb41, pRed_Cas9_recA_spoxb::rfp41 and pRed_Cas9_recA__lacZ41 were assembled using the modular assembly method.

\section{Strain and plasmid availability}

The plasmids used in this study are listed in Table 1. E. coli $\mathrm{DH} 5 \alpha$ was used as a cloning host, for fast performance, reaction mix assembled using the modular assembly method was directly transformed into $E$. coli MG1655. E. coli MG1655 was used in the genome engineering procedures. The coding sequences of all plasmids and modular parts developed here are physically available from the authors.

\section{Genome editing procedure}

A pair of $24 \mathrm{nt}$ oligos and a pair of $86 \mathrm{nt}$ oligos were designed to target genomic locus for editing. A simple DNA annealing procedure was used to obtain the N20 part and donor DNA part, which were assembled with Part1 and Part2 with a Golden Gate assembly reaction to the editing plasmid. Then the plasmid was transformed into E. coli, followed by plating on LB+ Kan $+1 \%(\mathrm{w} / \mathrm{v})$ glucose plate, and culturing at $30^{\circ} \mathrm{C}$. The resulting strain was grown in LB+ Kan for $2 \mathrm{~h}$ before $2 \mathrm{~g} / \mathrm{L}$ L-arabinose induction for Cas9 and gRNA expression. After culturing of $6 \mathrm{~h}$, the culture was plated on LB+ Kan $+\mathrm{L}$-arabinose agar plates, and the colonies were analyzed by colony PCR with a forward primer upstream of the left 
Table 1 The plasmids used for this work

\begin{tabular}{|c|c|c|}
\hline Plasmids & Relative characteristics & Resources \\
\hline pCas9 & Cas9 & {$[10]$} \\
\hline pKd46 & Exo, bet, gam, rep and arabinose operon & {$[3]$} \\
\hline pAra_Cas9__poxb300 & Arabinose operon, Cas9, gRNA with N20 and with homologous arms of about 300 bp for poxb & This work \\
\hline pRed_Cas9__poxB300 & $\begin{array}{l}\text { Derived from pKD46, exo, bet, gam, arabinose operon, Cas9, gRNA with N20 and with homologous arms } \\
\text { of about } 300 \text { bp for poxb deletion }\end{array}$ & This work \\
\hline pRed_Cas9__poxb::rfp300 & Derived from pRed_Cas9__poxB300, and with homologous arms of about 300 bp with rfp replace poxb & This work \\
\hline pRed_Cas9_spoxb100 & Derived from pRed_Cas9__poxB300, and with homologous arms of about 100 bp for poxb deletion & This work \\
\hline pRed_Cas9__poxb50 & Derived from pRed_Cas9__poxB300, and with homologous arms of about 50 bp for poxb deletion & This work \\
\hline pRed_Cas9_recA_spoxb300 & Derived from pRed_Cas9__poxB300,recA & This work \\
\hline pRed_Cas9_recA_spoxb41 & Derived from Red_Cas9_recA__poxb300, and with homologous arms of about 41 bp for poxb deletion & This work \\
\hline pRed_Cas9_recA_spoxb::rfp41 & Derived from Red_Cas9_recA__poxb300, and with homologous arms of about 41 bp for rfp replace poxb & This work \\
\hline pRed_Cas9_recA__lacZ41 & Derived from Red_Cas9_recA__poxb300, and with homologous arms of about 41 bp for lacZ deletion & This work \\
\hline
\end{tabular}

homology arm and a reverse primer downstream of the right homology arm. Colonies with expected PCR product were subjected to verification by DNA sequencing for further confirmation. At last, the temperature sensitive editing plasmid is cured by growing the edited strains at $37{ }^{\circ} \mathrm{C}$ overnight. A detailed genome editing protocol is provided in supplemental file (Additional file 1).

\section{Editing efficiency calculation}

For editing efficiency calculation, cell culture after induction process was diluted 100 fold, of which $20 \mu \mathrm{L}$ was plated on LB dishes for proximate colony counts. Colonies was screened by PCR and DNA sequencing to indentify correctly edited clones. With each experiment, 48 colonies are screened by colony PCR and the correct colonies were used for further verification by DNA sequencing. The editing efficiency equals the percentage of the correct colonies in all analyzed colonies. All experiments were repeated three times to obtain the means and standard variations.

When lacZ gene was targeted, cells were plated on IPTG and X-gal plates. The apparent editing efficiency was ratio of white colonies of total colonies. For further verification, white colonies were identified by colony PCR and DNA sequencing to obtain the real editing efficiency, which equaled ratio of the correctly edited colonies of total colonies.

\section{Result \\ CRISPR/Cas9 based chromosomal gene deletion of poxB with one plasmid construction and one transformation}

In this research we designed a genome editing method with one plasmid construction and one transformation. The CRISPR/Cas9 genomic editing system includes two parts, Cas9 and gRNA, simultaneous expression of which causes cleavage of bacterial genome, leaves blunt ends and causes cell death [19]. For this reason, Cas9 or gRNA should be repressed before genome editing event. In our preliminary studies, lac-derived promoter was unsuccessful as an inducible system for cas9 and gRNA expression due to its leaky expression. Thus, the glucose repressed, L-arabinose induced pBAD promoter was employed to express Cas9. With this strategy, the primitive oneplasmid system was designed to include functional parts of Cas9, gRNA and the temperature-sensitive replicon repA101ts [3]. The one plasmid editing procedure is illustrated in Fig. 1.

A non-essential gene poxB(GeneID: 946132) was selected as a target gene in E. coli MG1655 for genome editing [20]. An editing plasmid, pAra_Cas9__poxb300, was constructed to delete $513 \mathrm{bp}$ chromosomal fragment within poxB, which employed 300 bp homologous arms to recombine with the target locus. The plasmid was electroporated into E. coli MG1655 to initiate genome editing. After culturing and induction with $\mathrm{L}$-arabinose (Fig. 1), the culture was diluted 100 fold, of which $20 \mu \mathrm{L}$ were plated to obtain edited strains. A few hundred colonies were obtained. For the control experiment, cells were cultured without L-arabinose induction, thus, little Cas9 was expressed. The control group had many more colonies appeared on plate to form a bacterial meadow. This result demonstrated that Cas9 and gRNA were successfully expressed and functional. After arabinose induction, gRNA guided Cas9 to have killed most bacterial population that did not undergo poxB editing. By colony PCR and DNA sequencing, $20.8 \pm 8.8 \%$ random picked colonies from experiment group were determined to have been successfully edited, while no correctly edited ones from control group. This result demonstrated that our genome editing method was functional as expected. 


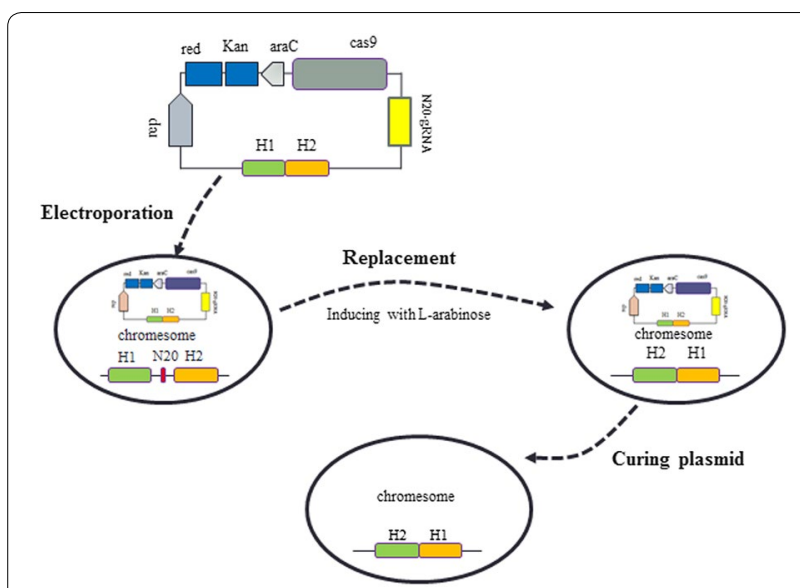

Fig. 1 The procedure of genome editing with one plasmid and one transformation. The first step is to assemble the editing plasmid. For the second step, the plasmid is transformed into E. coli, followed by plating on $\mathrm{LB}+\mathrm{Kan}+1 \%(\mathrm{w} / \mathrm{v})$ glucose plate, and grew at $30^{\circ} \mathrm{C}$. For the third step, the resulting strain is grown in $L B+$ Kan for $2 \mathrm{~h}$ before induction with $2 \mathrm{~g} / \mathrm{L}$ L-arabinose for Cas9 and gRNA expression. After culturing of $6 \mathrm{~h}$, the culture is plated on $\mathrm{LB}+\mathrm{Kan}+\mathrm{L}$-arabinose agar plates. Last step, the temperature sensitive editing plasmid is cured by growing the edited strains at $37^{\circ} \mathrm{C}$ overnight

\section{$\lambda$ Red recombinases improved editing efficiency}

To increase editing efficiency, we integrated $\lambda$ Red recombinases into the CRISPR/Cas9 plasmid, pRed_Cas9_ $\triangle p o x B 300$. This new one-plasmid system was tested to implement 513 bp chromosomal deletion of poxb gene with 300 bp homologous arms. Colonies from experimental group and control group were randomly picked for colony PCR identification with primer pairs pkd poxb_F and pkd_poxb_R. agarose gel electrophoresis of colony PCR was shown in Fig. 2a, that all experimental group colonies showed the expected shortened length after editing procedure. On the contrary, all colony PCR bands of control group showed original length. Sequencing data further proved that shortened bands were edited gene loci. Based on the results, although not all colonies were identified with PCR, $100 \%$ genome editing efficiency was achieved from randomly picked colonies. In addition, $1400 \mathrm{bp}$ chromosomal DNA of the poxB gene was also experimented to be deleted with $100 \%$ efficiency.

The one-plasmid genome editing system was then carried out for chromosomal gene replacement experiment. Plasmid pRed_Cas9__poxb::rfp300 was constructed for replacement of poxb with $r f p$ gene. In this study, $513 \mathrm{bp}$ of poxb was replacement by an $815 \mathrm{bp}$ fragment, which consisted of $r f p$ gene and SD sequence. A high editing rate of $100 \%$ was also obtained, part of the agarose gel electrophoresis of colony PCR was shown in Fig. $2 b$. The results demonstrated that with supplementation of $\lambda$ Red recombinases, the one plasmid strategy worked with $100 \%$ efficiency either with genomic knockout or replacement. And the target deletion length did not affect editing efficiency at least within 1500 bp range, which is long enough for normal gene knockout experiments.

\section{Genomic editing efficiency decreased with shorter homologous recombination arms}

To simplify plasmid construction, different length of homologous recombination arms were tested to find
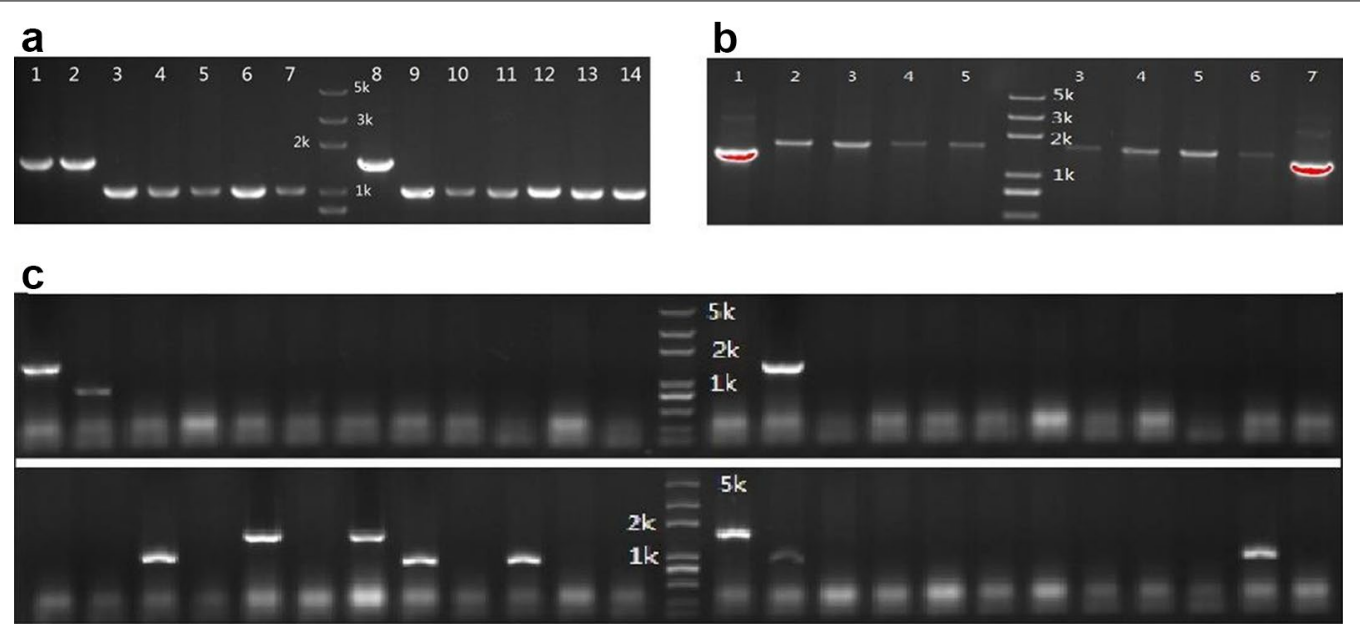

Fig. 2 Agarose gel electrophoresis of colony PCR for poxb editing. a poxb gene knock out with 513 bp deletion: lanes 1,2 and 8 were control group samples, others were experimental group samples. Colony PCR products of edited poxb gene were 1008 bp, and original were 1521 bp. b poxb replacement by $r f p$ : lanes 1 and 7 were control groups, others were experimental groups. Colony PCR products of edited poxb gene were 1823 bp, and original were 1521 bp. c poxb disruptions with homologous arms of 41 bp with both RecA and Red recombinases: all the bands were experimental group samples. Colony PCR products of edited poxb gene were 1008p 
the shortest that were functional. Homologous arms of around 300,100, and $50 \mathrm{bp}$ were chosen to test their editing efficiency. Plasmids pRed_Cas9__poxb100 and pRed_Cas9_Lpoxb50 were constructed to carry out the experiments. For each length, random colonies from the plates were identified with colony PCR. The results were illustrated in Table 2. With homologous arms of $101 \mathrm{bp}$ and $101 \mathrm{bp}$, the editing efficiency dropped to $69.3 \pm 1.17 \%$. And with 51 bp of homologous arms, we failed to obtain any successful edited clones from $50 \mathrm{col}-$ onies. This result indicated with current system, the editing efficiency declined rapidly with shorter homologous arms. Especially for the length of homologous arms less than $50 \mathrm{bp}$, the editing efficiency dropped to $0 \%$.

\section{Addition of recA enabled short homologous recombination arms for successful genomic editing}

For our one-plasmid genomic editing system, the most laborious part of work was plasmid construction. If the homologous arms were short enough, the two homologous arms could be assembled by primer embedding, instead of PCR and ligation. To simplify plasmid construction, it was important to make shorter homologs arms work for this method.

Reports have shown that recA improved red/ET recombination efficiency [21]. To increase editing efficiency, $r e c A$ gene was added to the one plasmid system to create pRed_Cas9_recA__poxb41 plasmid which carried $\lambda$ Red recombinases, CRISPR/Cas9 system, recA and two homologous arms of $41 \mathrm{bp}$. An editing efficiency of $13.8 \pm 7.9 \%$ was achieved in $E$. coli MG1655 with poxB gene deletion. Parts of the agarose gel electrophoresis of colony PCR is shown as Fig. 2c. For gene replacement, pRed_Cas9_recA_spoxb::rfp 41 was constructed for replacement of poxb with $r f p$ gene. With 41 bp homologous arms, an editing efficiency of $16.7 \pm 3.8 \%$ was achieved. The editing efficiency with 41 bp homologous arms is acceptable for genome editing experiment, since even with this efficiency, at least one successfully edited strain could be obtained from seven to eight colonies. Furthermore, colony PCR screening is not laborious and is to be performed even with high editing efficiency experiments.

Table 2 The editing efficiency and colony number at pox $B$ locus with different length of homologous arms

\begin{tabular}{llc}
\hline $\begin{array}{l}\text { Homologous arm } \\
\text { length }\end{array}$ & $\begin{array}{l}\text { Proximate colony } \\
\text { number }\end{array}$ & Editing efficiency \\
\hline $297 \mathrm{bp}$ plus $298 \mathrm{bp}$ & 500 & $100 \pm 0 \%$ \\
$101 \mathrm{bp}$ plus $101 \mathrm{bp}$ & 150 & $69.3 \pm 1.17 \%$ \\
$51 \mathrm{bp}$ plus $51 \mathrm{bp}$ & 50 & 0 \\
\hline
\end{tabular}

\section{One-plasmid method enabled convenient multi-round genome editing}

To demonstrate the application of one-plasmid system for multiple round editing, lacZ(Gene ID: 945006) gene was selected for target of the second round for the convenience of color indication [12]. After MG1655 1 poxb was obtained, editing plasmid pRed_Cas9_recA_ $\Delta p o x b 41$ was cured by growing colonies overnight at $37^{\circ} \mathrm{C}$. pRed Cas9_recA__lacZ41 was constructed to delete $1 \mathrm{~kb}$ of lacZ gene and transformed into MG1655 1 poxb. After induction process, the culture was diluted tenfold, of which $20 \mu \mathrm{L}$ were plated, and editing efficiency was calculated and showed in Table 3. Apparent efficiency represented percentage of white colonies in total colonies on plate, which showed phenotype of $\Delta l a c Z$. The editing efficiency was percentage of the colonies confirmed with PCR in total colonies. Interestingly, PCRs of some colonies turned out to have neither edited band nor original band, which was consistent with poxb editing situation. These colonies contributed to apparent edition efficiency but not editing efficiency. In addition, all the colonies verified for lac $Z$ editing were also subjected for colony PCR of poxb and confirmed to keep the poxb deletion. This result proved genomic stability of editing locus during multiple rounds by this method.

\section{Development of modularized assembly strategy for plasmid construction}

Using homologous arms of around $40 \mathrm{bp}$, we were able to design a rapid method of plasmid construction using a modular approach with Golden Gate assembly strategy [22]. The modularized plasmid (pRed_Cas9_recA_ $\Delta$ poxb41) was divided into four parts: part1, part2, N20 and donor DNA. The four parts were designed for each to carry a pair of 4 bp TypeII restriction enzyme linkers as illustrated in Fig. 3a, which were tested and optimized for best assembly efficiency.

Modular Part1 and part2 were the basic compositions for plasmid construction, which were ready made for editing of different loci, as showed in Fig. 3b. Part1 consisted of a resistance gene, an arabinose-inducible pBAD promoter with Cas9 and $\operatorname{rec} A$, a terminator, and a constitutive promoter for gRNA. Part2 consisted the temperature-sensitive replicon repA101ts, gRNA without N20 leading sequence, exo, bet, gam, rep and araC. N20 parts

Table 3 Efficiency of the two round consecutive editing with one-plasmid method

\begin{tabular}{lcc}
\hline & Apparent efficiency & Editing efficiency \\
\hline First round at poxB & Not determined & $13.8 \pm 7.9 \%$ \\
Second round at lacZ & $89.7 \pm 0.9 \%$ & $19.9 \pm 4.1 \%$ \\
\hline
\end{tabular}



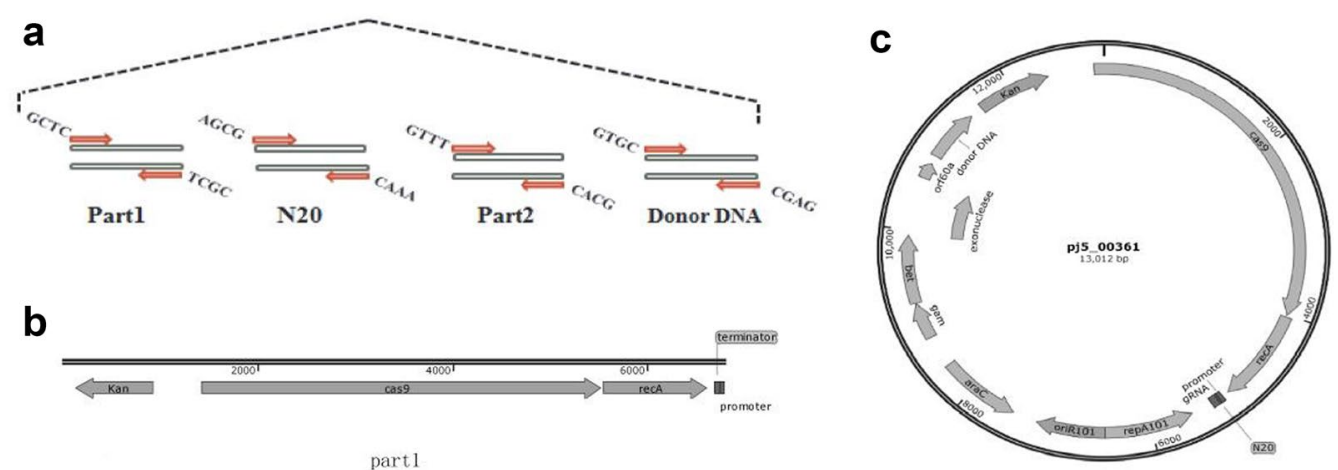

b
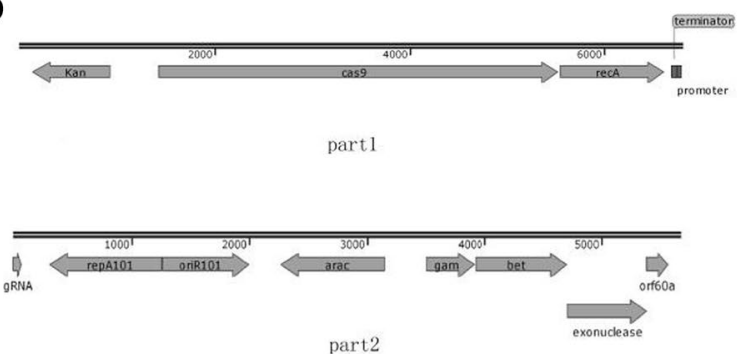

Fig. 3 Illustration of modularized assembly strategy. a Editing plasmid modular parts with optimized 4 nt TypellS resection enzyme linkers; $\mathbf{b}$ maps of fixed backbone modular part1 and part2; $\mathbf{c}$ map of assembled pRed_Cas9_recA__poxb300 from modular designed parts

and donor DNA parts were annealed from synthesized DNA oligos. A pair of $24 \mathrm{nt}$ oligos and a pair of $86 \mathrm{nt}$ oligos were needed for editing of one locus. Only a simple DNA annealing experiment and a Golden Gate assembly experiment were performed for the plasmid construction, without any necessities for PCR, DNA purification, or gel electrophoresis. The construction could be completed within four hours with minimal lab work. The assembled plasmid from these 4 parts was illustrated in Fig. 3c.

\section{The development of genomic DNA editing protocol with one plasmid construction and one transformation}

To develop a mature protocol for fellow researchers to use without further try and error, we went through the experiment process several times and optimized parameters to ensure successful genome editing. The protocol was divided in 4 steps. The first and second steps were to construct two modular parts, N20 part and donor DNA part. The third step was to assemble two readymade modular parts with N20 and donor DNA parts with Golden Gate method in one reaction. The last step included one transformation and one culturing practice to obtain edited strains. The detailed protocol is presented in Additional file 1.

\section{Discussion}

Compared to other CRISPR-based genome modification system, our one-plasmid system has the advantage of fast and easy operation with high efficiency. A simple comparison is summarized in Table 4 to illustrate difference of this technique compared with several recently developed methods. Information in this table is by no means accurate and subjective, and some description is by estimation. Due to the simplicity of one plasmid without any other supplemental DNA material, the experiment process is simple and less time consuming than other methods, only takes 3 days to complete. Also, labor intensity is relatively low, since major work only includes one plasmid construction and one transformation plus colony screening for editing plasmid and edited strains. We have experimented thoroughly to have selected optimized plasmid assembly strategy with ready-made parts (part1 and part2) and fixed linkers for editing plasmid construction. Because of this modularized design of plasmid construction, plasmid construction becomes very easy and economical. The construction process only takes $4 \mathrm{~h}$ after receiving synthesized DNA oligos, and minimal sets of oligos ensure low cost. The preferred editing protocol employed short homologous arms to achieve easiest plasmid construction but sacrificed some editing efficiency. In this comparison category, our method might have no advantage comparing to others (Table 4). However, at least one successfully edited strain could be obtained from seven to eight colonies. Furthermore, colony PCR screening is not laborious and is to be performed even with high editing efficiency experiments.

The method also has an advantage for editing of multiple loci. The system requires only one temperature sensitive plasmid, which makes plasmid curing process easy and fast. In continuous process with our technique, $\mathrm{X}$ loci could be edited within $3 \times$ days. This rapid and simple method for $E$. coli genome editing has already been used in our research group, for example editing promoters 


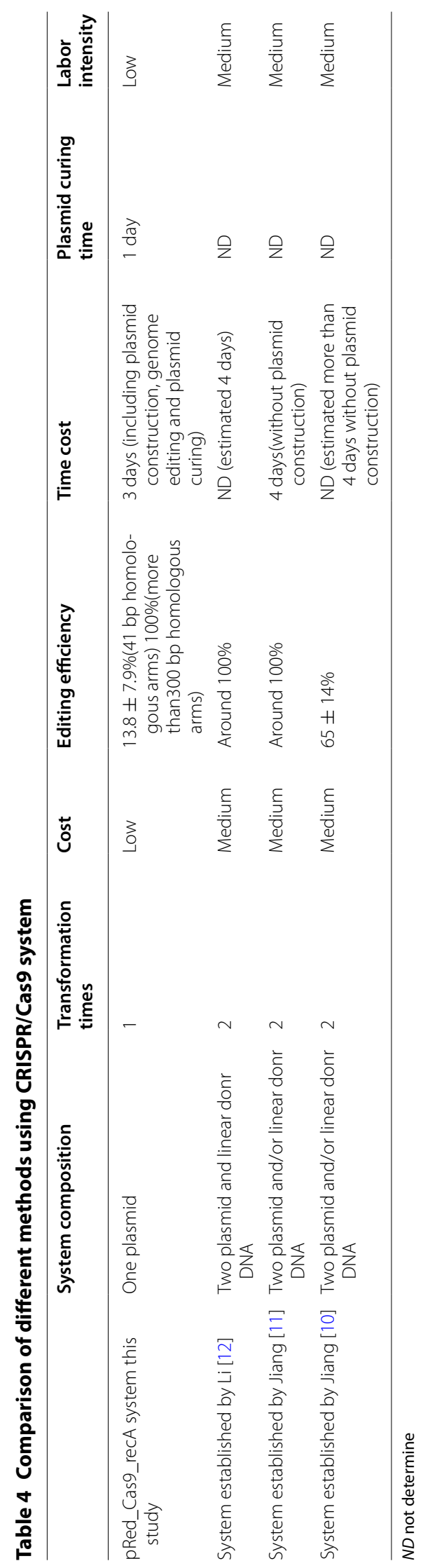


of $c r t$, idi and $d x s$ in QL002 strain [23]. And it has also already been adopted by other labs in our institute.

High throughput and automatic genomic editing is one highly desired technique by molecular biologists [24, 25]. Due to its modular design, this method could be applied to develop automatic genome editing techniques. In plasmid construction process, DNA annealing and Golden Gate assembly process could be easily transformed to computer controlled practice with a liquid handler platform [26, 27]. Plasmid transformation and induction process could be automated as the MAGE system [24, 28]. Colonies PCR screening process can also be performed by automatic system [26, 27]. We expect a combined automatic system to be able to perform high-throughput, rationally designed genome editing based on our methods.

Colony PCR was used to identify successfully edited clones based on the size of PCR products in this work. However, as shown in Fig. 2c, some colony PCRs turned out to have neither edited band nor original band, just empty lanes. These colony PCRs were repeated with more sets of primers and were confirmed not to be able to obtain amplification products. A newly published article suggested that $E$. coli strains might possess a distinct end-joining activity that repairs double strand breaks (DSBs) and generate genome rearrangements. This mechanism, named alternative end-joining (A-EJ), is characterized by extensive DNA end restriction and large chromosomal loss [29]. We hypothesized that the colonies without $\mathrm{PCR}$ products might be $E$. coli cells that survived CRISPR mediated DSBs by A-EJ and underwent large DNA deletion. This hypothesis was partially proved by editing of lac $Z$ locus. As illustrated in Table 3, 89\% edited strains were white which apparently had lac $Z$ knocked out. However, only $19 \%$ was confirmed to have shortened lacZ gel bands by colony PCR, which was the indication of precise editing as designed. Others showed no band or smear on gel, indicating changed chromosomal status around the lac $Z$ locus. To analyze a larger area, primers targeting a region up to $7.5 \mathrm{~kb}$ around the Cas 9 cleavage locus were designed and applied. However, no target PCR products were obtained with these primers either. Due to cost reason, we had not sequence these strains yet to find out their genomic changes. According to a newly published article, our result suggest CRISPR mediated DSBs may induce A-EJ repair in E. coli, and cause very large genomic deletion or rearrangement [30].

The method established in this paper has several advantages compared to others, however, its editing efficiency for short homologous arms did not reach $100 \%$. There are some strategies we would exploit to improve in the near future. For example, using new CRISPR proteins, such as Cpf1 might help to improve recombination frequency by its different cleavage pattern [31]. Moreover, we might further improve homologous recombination efficiency by experimenting with more recombinases from various sources [32, 33].

\section{Conclusion}

In this study, we achieved the goal of development of a very fast and easy genome editing technique with high efficiency based on CRISPR/Cas9 system that only required the work of one plasmid construction and one transformation, which allowed modification of a chromosome locus within 3 days and could be performed continuously for multiple loci.

\section{Additional files}

Additional file 1. Standard genome editing protocol developed in this research.

Additional file 2. Plasmids profiles and sequences

\section{Authors' contributions}

$C B, X Z$ and DZ conceived the experiments. DZ, SY, BX, HS, LY and JL performed the experiments. $C B$ and $D Z$ analyzed the results. $C B$ and $D Z$ wrote the paper. All authors read and approved the final manuscript.

\section{Author details}

${ }^{1}$ Tianjin Institute of Industrial Biotechnology, Chinese Academy of Sciences, Tianjin 300308, China. ${ }^{2}$ Key Laboratory of Systems Microbial Biotechnology, Tianjin Institute of Industrial Biotechnology, Chinese Academy of Sciences, 32 West 7th Ave, Tianjin Airport Economic Park, Tianjin 300308, China.

\section{Acknowledgements}

This research was financially supported by the National High Technology Research and Development Program of China (2015AA020202), Tianjin Key Technology R\&D program of Tianjin Municipal Science and Technology Commission (Y5M2121111) and Novo Nordisk-Chinese Academy of Sciences (NN-CAS) Research Fund (NNCAS-2015-2).

\section{Competing interests}

The authors declare that they have no competing interests.

\section{Availability of data and materials}

We provide support if necessary data for publication of the article, Supporting data is provided in Additional file 1, plasmids profiles and sequences is provided in Additional file 2.

\section{Funding}

This research was supported by the National High Technology Research and Development Program of China (2015AA020202), Tianjin Research Program of Application Foundation and Advanced Technology (15JCYBJC24200) and Novo Nordisk-Chinese Academy of Sciences (NN-CAS) Research Fund (NNCAS-2015-2).

Received: 1 October 2016 Accepted: 25 November 2016

Published online: 01 December 2016

\section{References}

1. Yu D, Ellis HM, Lee E-C, Jenkins NA, Copeland NG. An efficient recombination system for chromosome engineering in Escherichia coli. Proc Natl Acad Sci USA. 2000;97(11):5978-83. 
2. Jantama K, Haupt M, Svoronos SA, Zhang X, Moore J, Shanmugam K, Ingram L. Combining metabolic engineering and metabolic evolution to develop nonrecombinant strains of Escherichia coli $\mathrm{C}$ that produce succinate and malate. Biotechnol Bioeng. 2008;99(5):1140-53.

3. Datsenko KA, Wanner BL. One-step inactivation of chromosomal genes in Escherichia coli K-12 using PCR products. Proc Natl Acad Sci USA. 2000;97(12):6640-5.

4. Bibikova M, Golic M, Golic KG, Carroll D. Targeted chromosomal cleavage and mutagenesis in Drosophila using zinc-finger nucleases. Genetics. 2002;161(3):1169-75.

5. Miller JC, Tan S, Qiao G, Barlow KA, Wang J, Xia DF, Meng X, Paschon DE, Leung E, Hinkley SJ. A TALE nuclease architecture for efficient genome editing. Nat Biotechnol. 2011;29(2):143-8.

6. Mahfouz MM, Li L, Shamimuzzaman M, Wibowo A, Fang X, Zhu J-K. De novo-engineered transcription activator-like effector (TALE) hybrid nuclease with novel DNA binding specificity creates double-strand breaks. Proc Natl Acad Sci USA. 2011;108(6):2623-8.

7. Horvath P, Barrangou R. CRISPR/Cas, the immune system of bacteria and archaea. Science. 2010;327(5962):167-70.

8. Jakočiūnas T, Bonde I, Herrgård M, Harrison SJ, Kristensen M, Pedersen LE, Jensen MK, Keasling JD. Multiplex metabolic pathway engineering using CRISPR/Cas9 in Saccharomyces cerevisiae. Metab Eng. 2015;28:213-22.

9. Jinek M, Chylinski K, Fonfara I, Hauer M, Doudna JA, Charpentier E. A programmable dual-RNA-guided DNA endonuclease in adaptive bacterial immunity. Science. 2012;337(6096):816-21.

10. Jiang W, Bikard D, Cox D, Zhang F, Marraffini LA. RNA-guided editing of bacterial genomes using CRISPR-Cas systems. Nat Biotechnol. 2013;31(3):233-9.

11. Jiang Y, Chen B, Duan C, Sun B, Yang J, Yang S. Multigene editing in the Escherichia coli genome via the CRISPR-Cas9 system. Appl Environ Microbiol. 2015;81(7):2506-14.

12. Li Y, Lin Z, Huang C, Zhang Y, Wang Z, Tang YJ, Chen T, Zhao X. Metabolic engineering of Escherichia coli using CRISPR-Cas9 meditated genome editing. Metab Eng. 2015;31:13-21.

13. Shalem O, Sanjana NE, Hartenian E, Shi X, Scott DA, Mikkelsen TS, Heckl D, Ebert BL, Root DE, Doench JG. Genome-scale CRISPR-Cas9 knockout screening in human cells. Science. 2014;343(6166):84-7.

14. Fujii W, Kawasaki K, Sugiura K, Naito K. Efficient generation of large-scale genome-modified mice using gRNA and CAS9 endonuclease. Nucleic Acids Res. 2013;41(20):e187.

15. Chang N, Sun C, Gao L, Zhu D, Xu X, Zhu X, Xiong J-W, Xi JJ. Genome editing with RNA-guided Cas9 nuclease in zebrafish embryos. Cell Res. 2013;23(4):465-72.

16. Pyne ME, Moo-Young M, Chung DA, Chou CP. Coupling the CRISPR/Cas9 system to lambda Red recombineering enables simplified chromosomal gene replacement in Escherichia coli. Appl Environ Microbiol. 2015;81(15):5103-14.
17. Benzinger R, Enquist LW, Skalka A. Transfection of Escherichia coli spheroplasts. V. Activity of recBC nuclease in rec+ and rec minus spheroplasts measured with different forms of bacteriophage DNA. J Virol. 1975;15(4):861-71.

18. Hillson NJ, Rosengarten RD, Keasling JD. j5 DNA assembly design automation software. ACS Synth Biol. 2011;1(1):14-21.

19. Ronda C, Pedersen LE, Sommer MO, Nielsen AT. CRMAGE: CRISPR optimized MAGE recombineering. Sci Rep. 2016;6:19452.

20. De Mey M, De Maeseneire S, Soetaert W, Vandamme E. Minimizing acetate formation in E. coli fermentations. J Ind Microbiol Biotechnol. 2007:34(11):689-700.

21. Wang J, Sarov M, Rientjes J, Hu J, Hollak H, Kranz H, Xie Y, Stewart AF, Zhang Y. An improved recombineering approach by adding RecA to $\lambda$ red recombination. Mol Biotechnol. 2006;32(1):43-53.

22. Engler C, Kandzia R, Marillonnet S. A one pot, one step, precision cloning method with high throughput capability. PLoS ONE. 2008;3(11):e3647.

23. Zhao J, Li Q, Sun T, Zhu X, Xu H, Tang J, Zhang X, Ma Y. Engineering central metabolic modules of Escherichia coli for improving $\beta$-carotene production. Metab Eng. 2013;17:42-50.

24. Wang HH, Isaacs FJ, Carr PA, Sun ZZ, Xu G, Forest CR, Church GM. Programming cells by multiplex genome engineering and accelerated evolution. Nature. 2009:460(7257):894-8.

25. Jakočiūnas T, Jensen MK, Keasling JD. CRISPR/Cas9 advances engineering of microbial cell factories. Metab Eng. 2016;34:44-59.

26. Linshiz G, Stawski N, Poust S, Bi C, Keasling JD, Hillson NJ. PaR-PaR laboratory automation platform. ACS Synth Biol. 2012;2(5):216-22.

27. Linshiz G, Stawski N, Goyal G, Bi C, Poust S, Sharma M, Mutalik V, Keasling JD, Hillson NJ. PR-PR: cross-platform laboratory automation system. ACS Synth Biol. 2014;3(8):515-24.

28. Wang HH, Kim H, Cong L, Jeong J, Bang D, Church GM. Genomescale promoter engineering by coselection MAGE. Nat Methods. 2012;9(6):591-3.

29. Chayot R, Montagne B, Mazel D, Ricchetti M. An end-joining repair mechanism in Escherichia coli. Proc Natl Acad Sci USA. 2010;107(5):2141-6.

30. Cui L, David B. Consequences of Cas 9 cleavage in the chromosome of Escherichia coli. Nucleic Acids Res. 2016;44(9):4243-51.

31. Zetsche B, Gootenberg JS, Abudayyeh $\mathrm{OO}$, Slaymaker IM, Makarova KS, Essletzbichler P, Volz SE, Joung J, van der Oost J, Regev A. Cpf1 is a single RNA-guided endonuclease of a class 2 CRISPR-Cas system. Cell. 2015:163(3):759-71.

32. Dudáš A, Chovanec M. DNA double-strand break repair by homologous recombination. Mutat Res Rev Mut Res. 2004;566(2):131-67.

33. Court DL, Sawitzke JA, Thomason LC. Genetic Engineering Using Homologous Recombination 1. Annu Rev Genet. 2002;36(1):361-88.

\section{Submit your next manuscript to BioMed Central and we will help you at every step:}

- We accept pre-submission inquiries

- Our selector tool helps you to find the most relevant journal

- We provide round the clock customer support

- Convenient online submission

- Thorough peer review

- Inclusion in PubMed and all major indexing services

- Maximum visibility for your research

Submit your manuscript at www.biomedcentral.com/submit
BioMed Central 